\title{
AN INNOVATIVE MULTI-CRITERIA DECISION METHOD FOR IMPACTS'ASSESSMENT OF UNESCO BRAND
}

\author{
Paola Boati \\ Ph.D at Politecnico di Torino \\ Torino, ITALY \\ E-mail: p.boati@virgilio.it
}

\begin{abstract}
The UNESCO World Heritage List was created to identify unique and authentic sites from a cultural or natural point of view, whose protection and conservation are important for the entire world community and to which is given an "outstanding universal value". So the World Heritage Status has more and more became an opportunity not only as witness of historical, aesthetic and identity values, but also to use the brand as a tool for achieving a sustainable local development of the whole context.

In fact, the inscription on the List brings positive effects on the surrounding context and can create significant impacts also from the environmental, occupational and socio-economic point of view. However, it also brings to light negative aspects, first and foremost a high influx of visitors, due to visibility of the site at an international level, with the consequent damage to the environment and the exclusion of local activities by gains related to the tourism sector.

The purpose of this paper is to investigate the role that the inclusion of a site on the UNESCO WHL can have for the creation of local development and sustainability processes within the territory in which the property is inserted, using and mixing different economic valuation techniques to identify and quantify impacts and effects that are created on the context of the site, on local actors involved in its management, visitor and resident population. The methodology is based on a wide literature review, the comparison of statistical data regarding some Italian cities, some included in the List and other not equipped with this brand and finally the setup of an innovative framework of evaluation, objective and scientifically robust, able to determine, identify and weigh the impacts resulting from the inscription of a site on the UNESCO WHL.
\end{abstract}

Keywords: UNESCO, impact assessment, integration and innovation. 
ISAHP Article: Boati/An innovative multi-criteria decision method for impacts' assessment of UNESCO brand. International Symposium of the Analytic Hierarchy Process 2014, Washington D.C., U.S.A.

\section{Introduction}

UNESCO - United Nations, Educational, Scientific and Cultural Organization - was founded November 16, 1945 in London, to encourage the collaboration among nations in the fields of science, education, culture and communication. For over 60 years, UNESCO is recognized as the only international organization with a specific mandate in the field of culture, and in this capacity has always been a leading advocate for the strengthening of the role of culture in local development. With the intention to preserve and promote cultural and natural heritage, was created the World Heritage List (WHL), with the aim of identifying and protecting internationally some sites of "outstanding universal value": inclusion in this list over the years has proved to be, for the sites involved, a powerful aid to local economic growth, social development and poverty eradication.

However, the only protection and conservation of cultural and environmental heritage are not sufficient; the valuation of these assets also stems from their ability to create and promote local development processes, from the economic, social and environmental points of view. The inclusion of a site in the UNESCO WHL establishes the worldwide recognition of the importance of these heritage and is also an important moment of reflection and analysis of real opportunities for sustainable development in order to involve local resources in integrated actions of protection, conservation and territorial enhancement.

It is appropriate, therefore, to identify and quantify, at local level, the effects that are created by the inclusion of a site on the World Heritage List, in order to better manage the resulting opportunities and mitigate any weaknesses, to determine if indeed this important brand brings benefits in the surrounding territory and may be an opportunity to implement policies and actions of sustainable and durable development.

These concepts indicates the need to investigate more thoroughly the relationships and processes that link the recognition of UNESCO World Heritage Site and local development of the surrounding area. So, because of in recent years we are witnessing a greater demand for inclusion in the World Heritage List, can this international recognition promote sustainable development of the local context to which the site belongs?

\section{Literature Review}

In recent years, more and more studies are working to determine the possible direct and indirect benefits in the socio-economic field related to protected heritage, whether natural or cultural. Researchers have begun to focus on the economic impact of cultural heritage, such as measuring the impact of regeneration on jobs and household income, the role of the historic buildings as incubators for small businesses, the incremental impact of cultural tourism, the contribution of heritage conservation in the revitalization of historic city centers and in the influences which may have the antique details on property values.

Among the various documents analyzed for the framing of the "problem", below are analyzed those most specific, inherent with issues related to the relationship between UNESCO brand and local development.

A study concerning the potential socio-economic impacts for sites published in 2009 by the UNESCO World Heritage Lake District Project (Scotland), shows that the costs of obtaining the WHL inscription have increased dramatically over the past ten International Symposium of 2 Washington, D. C. the Analytic Hierarchy June 29 - July 2, 2014 
ISAHP Article: Boati/An innovative multi-criteria decision method for impacts' assessment of UNESCO brand. International Symposium of the Analytic Hierarchy Process 2014, Washington D.C., U.S.A.

years, precisely because of the raising up of the number of sites that require it. The increase in costs has led many development agencies to question whether the investments made are justified by a real economic return resulting from the inclusion of the site in the WHL.

Sieber Jula-Kim (2010), wonders how historic towns can receive benefits by the inclusion in the WHL and at the same time avoid becoming a "Walt Disney" for tourists. In many places, in fact, there are fears that the brand can be used primarily to start a marketing campaign with capitalistic purposes only. The requirement for a rapid return on the investment could lead to a too fast process of development and non-use of best practices for sustainability. Even the frequent restoration work, with the reconstruction of some important buildings, can lead to the creation of "stages" to be used by tourists for their photos and the proposition of artificial environments and totally alien to the local context in which they appear. "Throughout the world there is a risk that the UNESCO sites are being transformed into open-air museums, theme parks or tourist attractions but only a few bring revenue for local people and many changes on the urban context in which they live".

According to Van der Aa (2005), UNESCO recognition represents a possible tool for better preservation of cultural heritage, but does not provide a definitive guarantee even after the registration. It is therefore necessary that the inscription of a site in the WHL may lead to collaboration between different stakeholders in order to gain support of various scales and levels of action.

According to Prud'homme (2008), the greater visibility and reputation worldwide of UNESCO sites and the consequent flow of tourists can create conditions to improve but also negative consequences. The determination of the effects that are created at local level from the inclusion of a site in the WHL is a necessary operation to manage the opportunities and mitigate any weaknesses, identifying main impacts arising from increase in tourism and the funding provided for the conservation of cultural heritage. By analyzing in detail the studies, it is clear that "actual impacts on local development result from the inclusion of a site in the WHL are largely overstated. In summary, the direct link between membership in the WHL and local economic development is probably quite limited."

By analyzing the various documents and studies, it was found that the inscription on the WHL is definitely a favorable factor for development, but by itself it is neither necessary nor sufficient to ensure the proper conservation and enhancement of the sites involved. The increase of tourists is mainly due to the spread visibility of the site on an international level, however, by the time subsequent to the designation, each location is handled in different ways, without obtaining, in many cases, new processes of regeneration and socio-economic exploitation by one development of the largest and most comprehensive site and its local context.

\section{Objectives}

The aim of this research is to investigate the role that the inclusion of a site in the UNESCO WHL can play for the creation of local development and sustainability processes within the territory in which the site is inserted, using different economic evaluation techniques to identify and quantify impacts and effects that are created on the context surrounding the site, on local actors involved in its management, visitor and resident population. 
ISAHP Article: Boati/An innovative multi-criteria decision method for impacts' assessment of UNESCO brand. International Symposium of the Analytic Hierarchy Process 2014, Washington D.C., U.S.A.

The objectives of this research arises are different:

1. A first objective is to verify if the inscription of a site on the World Heritage List of UNESCO plays an important role in economic development, and especially tourism and economic, compared to other destinations which do not have such international recognition.

2. The second objective relates to the applicative use of some economic valuation techniques for the definition of an appropriate support system.

3. The third objective involves the identification of the impacts deriving from the inclusion of a site on the World Heritage List of UNESCO.

\section{Research Design/Methodology}

UNESCO has always recognized the importance and the value of Italian cultural heritage, so much so that our country has the largest number of Sites in the WHL. Just the UNESCO brand can be seen as a form of recognition of the value and warranty for cultural heritage that can make a difference for the creation of best practices for sustainable local development and shared with the resident population, the prospect of an improvement in economic conditions, employment, social and cultural rights. In addition, considering that the increasing "race" to grab this international recognition sees the presence of numerous competitors, it is appropriate to investigate whether the increased visibility and reputation in the world of the UNESCO sites can create an effective and desirable improvement of the conditions or adverse consequences.

The main areas of the research are:

- The World Heritage sites and effects on cultural tourism.

- The World Heritage sites and the effects on local sustainable development. What are the effects resulting from the inclusion of a site on the World Heritage List?

The methodological framework developed for this study involves a combination of statistical data, Community Impact Evaluation (CIE) and Analytic Network Process (ANP), allowing the integration of the econometric analysis with the identification of impacts for different sectors of the community and with the determination of the most viable alternatives, in order to combine the priorities of the stakeholders and the different aspects related to the sustainable development of a local tourist destination.

The framework lays its foundations on the literature review on the relationship between the UNESCO brand, local development and evaluations techniques for cultural heritage.

Case study are referred to three Italian cities, Venaria Reale and Verona included in the UNESCO WHK, and Lucca, not yet recognized as World Heritage.

The construction of the valuation model is composed of 3 main phases: 1. Creating a set of statistical indicators by analyzing and comparing econometric data, for an objective and detailed framework, able to include all the aspects related to cultural heritage and local development;

2. Structuring a matrix of impacts related to the sectors of the community identified through the adaptation and application of the CIE. In this phase, the statistical indicators identified in the previous phase, are expressed through qualitative judgments (+ or -) and referred to various categories of impacts, financial, economic, social, cultural and environmental; 
ISAHP Article: Boati/An innovative multi-criteria decision method for impacts' assessment of UNESCO brand. International Symposium of the Analytic Hierarchy Process 2014, Washington D.C., U.S.A.

3. Identification of better alternatives through the use of ANP and the construction of a model containing the clusters of impacts, alternatives and sectors of the community (identified and evaluated in the previous step) and the pairwise comparison between the internal nodes.

The framework used for the evaluation of the impacts that the UNESCO brand can produce, provides that the valuation techniques are analyzed, adapted and integrated in order to be applied to case studies, with the intention of creating a new assessment tool for cultural heritage and local development.

At the end of the application of the valuation method identified, it will get a list of alternatives related to every sector of the community, which will lead to the final classification of the site (UNESCO or not) where the impacts results most positive (through the classification of related indicators).

\section{Data/Model Analysis}

The assessment method resulting from the revision and adaptation of the CIE technique was further supported by the application of the Analytic Network Process (ANP), which allows comparability of targets heterogeneous, often expressed in different units, and connected to each other by various dependencies. Completing and integrating the CIE with the ANP technique, in addition to identifying the impacts (positive and negative) for each sector of the community, it is possible to determine, through the pairwise comparison, the classification of the categories of impacts reported for each alternative identified as case study, in order to reach an overall assessment of best alternatives and the recognition of the role that UNESCO can play in the activation of local development in the context of reference. The composition of the model is based on the creation of a structured network, which "presupposes the existence of a hierarchy of control which gives rise to subnetworks in which the groups are contained, the elements and alternatives." (Bottero et al., 2008 ). In this way, each subnet can evaluate separately the various elements which are then integrated at a later date for the final determination of preferability ranking of the alternatives.

The application of the method is carried out through the determination of the main objective, which in turn is connected with the cluster of the sectors of the community (identified thanks to the use of the CIE). Internal nodes in this cluster have been identified with different types of Community sectors (people living, working, visiting, governing).

The application of the ANP in structured network was carried out by using the software "Super Decisions". The main objective of the model is connected with the cluster of sectors of the community, which contains inside subnets for people living, working, visiting and governing. Within this subnetwork are contained clusters of impacts, with the nodes related to the financial, economic, social, cultural, environmental and clusters of alternatives, with the nodes of individual case study (Venaria, Verona and Lucca). 
ISAHP Article: Boati/An innovative multi-criteria decision method for impacts' assessment of UNESCO brand. International Symposium of the Analytic Hierarchy Process 2014, Washington D.C., U.S.A.

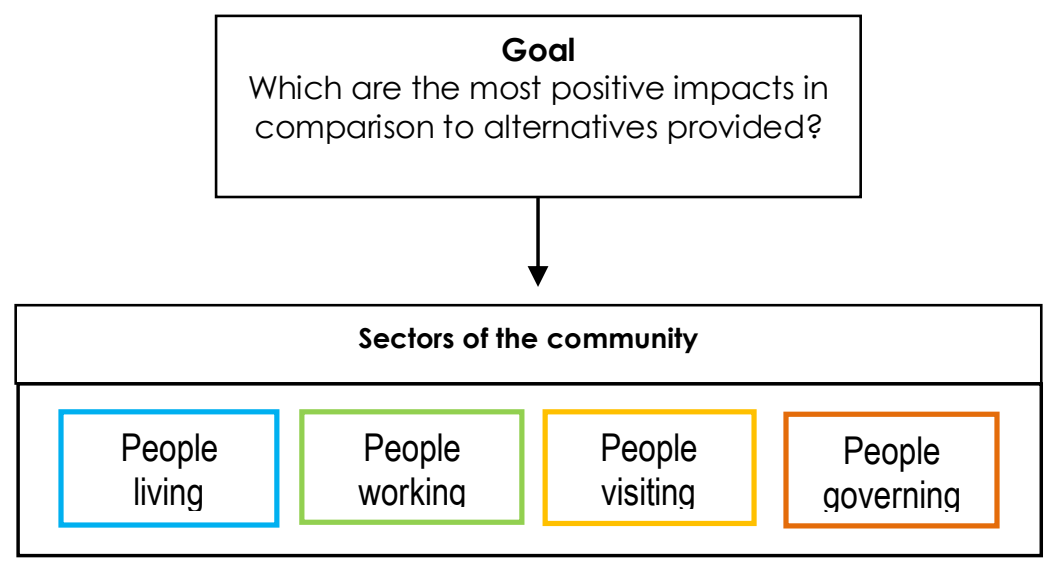

Each node referred to any sector of the community contains subnets. Within each subnetwork are inserted cluster impacts, with the nodes related to the financial, economic, social, cultural, environmental, and clusters of alternatives, including the nodes of the individual cities considered as a case study.

Through the application of the ANP is possible to identify a classification of cities and impacts, having identified them as positive or negative by CIE, and giving them an order of preferability.

The final model for each sector of the community, provides the explanation of the impacts through statistical indicators identified in the analytical framework and their comparison with the alternatives provided, that individual cities taken as a case study:

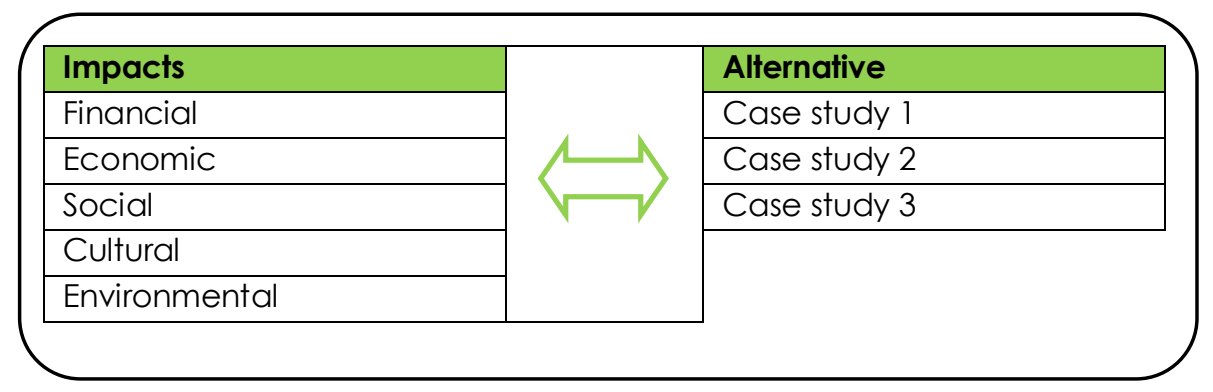

The dependency relationships between these two clusters have been provided in order to determine two-way, through a pairwise comparison, the priority and best impacts referred to each single alternative and to make value judgments that lead to the identification of the better alternative. At the end of the application of the valuation method identified, then you will get a list of alternatives related to every sector of the community, which will lead to the final classification of the case study where the impacts results most positive (through the classification of related indicators).

The phase of the evaluation is to compare the individual nodes of each cluster so as to give value judgments on each item through the pairwise comparison. The judgments of preference were expressed with a positive numeric value, from 1 to 9 .

In order to assign weights to the different cluster, you must perform pairwise comparisons between the various network elements and assign them different weights. Initially you are asked for any alternative proposal, what kind of impact is preferable over another, in this case the priority derived from the results of the assessment carried through the Community Impact Evaluation. 
ISAHP Article: Boati/An innovative multi-criteria decision method for impacts' assessment of UNESCO brand. International Symposium of the Analytic Hierarchy Process 2014, Washington D.C., U.S.A.

With the aim to make a more precise search, not only are valued dependencies among alternatives and impacts, but the impacts themselves were evaluated from the point of view of the alternatives.

By application of ANP to the case studies examined, it was found that Lucca is the most preferred alternative, followed by Verona and Venaria Reale. In this case therefore the impacts related to the presence of tourists and the consequent effects on local development are generally more positive and significant in the Tuscan city than the other two cities analyzed as a case study.

The results obtained through the application of the valuation method to the case studies considered may seem unexpected. It would be shown that the inclusion of a site in the UNESCO World Heritage List does not have a significant added value from the socio-economic development point of view of the local context.

\section{Limitations}

The main limitations that this research may have mainly consist of a "critical" to the valuation method used. The number and choice of case study is a limitation that this research might have. In fact, even if the evaluation survey was addressed to the analysis of the environment and its characteristics in a comprehensive manner, it remains to verify the general validity of the results, also considering other locations, perhaps in an international context.

The Analytic Network Process (ANP) has made it possible to implement complex assessments and complete with the ability to have relationships of dependence, feedback and pairwise comparisons within the valuation model. Nevertheless precisely the complexity of the model led to the definition of several pairwise comparisons and the time that the evaluator must employ to carry out the assessment is consistent. In addition, when you were to add to a model or an alternative criterion, the results of the final list definitely would change. In this regard, the most important problem could be the assignment of weights to different clusters that make up the model. The weights that are assigned should reflect the relative importance of individual indicators, and in this study the application of the CIE was of great importance in order to provide a proper weight to different elements of the model.

\section{Conclusions}

The application of the proposed method in the ex post phase has allowed to develop an innovative tool to frame the complexity of the aspects that make up the local development of a cultural and tourist destination, providing a possible greater effectiveness in the identification and management of impacts for different local actors involved. The results obtained lead to the conclusion that, although the UNESCO status and the inclusion of a site in the WHL may become a symbol of quality for the whole territory, is not always necessary to get this recognition in order to generate positive economic impacts on the local context, because it is the latter that determines the true capacity of a territory to be competitive and attract visitors and investments.

In conclusion, this study has allowed the application of the evaluation system to determine what role and the impacts deriving from the inscription in the UNESCO World Heritage List compared to other locations that are not equipped with the brand. Joint application of statistical analysis, Community Impact Evaluation and Analytic Network Process has been possible to develop an evaluation method that proceeds by distinct but interdependent phases. In the case of analysis characterized by numerous 
ISAHP Article: Boati/An innovative multi-criteria decision method for impacts' assessment of UNESCO brand. International Symposium of the Analytic Hierarchy Process 2014, Washington D.C., U.S.A.

data and complex aspects, the use of this methodology and techniques combined together can be justified by their contributions that these make for the understanding and definition of the basic question, since each of the three phases identified in the valuation framework is dependent on the other two in order to obtain a complete and comprehensive methodology for evaluation of the various aspects that make up the basic problem analysis. "Innovation " as suggested in this research lies not so much in the application of valuation methodologies, as well as in their use in the ex post stage, enabling you to improve the measurement and monitoring of impacts occurring in terms of sustainability strategies and policies put taking place in cultural tourism destinations to achieve local development objectives.

From the application "large-scale" method of evaluation identified it would be possible to get an overview of the largest and most comprehensive, analyzing the data and dynamics implemented in several Italian cities that enjoy recognition in the UNESCO World Heritage Site.

In addition, the results obtained would be possible to create a "handbook" of good practices found in the cities analyzed as case studies and a set of guidelines containing strategies and guidelines to be put in place to achieve them. From the results obtained through the ex-post analysis of the experiences of conservation and enhancement of cultural and environmental heritage you would be able to identify the key steps that wealth should follow the processes, and the assessment of the impacts and possible effects on the local environment, the path identified could be seen as a new method for evaluating and defining strategies and targeted interventions towards sustainable development and lasting affecting both sites already falling in the circuit UNESCO, and the destinations that are preparing to submit the required application.

\section{Key References}

Lichfield N., (1996). Community Impact Evaluation. London: UCL Press

Saaty, T.L., \& Peniwati, K. (2007). Group decision-making: Drawing out and reconciling differences. Pittsburgh, PA: RWS Publications.

Bottero M., Lami I.M, Lombardi P, (2008) Analytic Network Process - Applicazioni per la valutazione di scenari di trasformazione urbana e territoriale, Firenze: Alinea

Brandon P.S, Lombardi P., (2011)., Evaluating Sustainable Development in the Built Environment. II Edition, Wiley-Blackwell (GBR) 